This is the author's final version of the contribution published as:

[Barge P., Gay P., Merlino V., Tortia C., Radio frequency identification technologies for livestock management and meat supply chain traceability, Canadian Journal of Animal Science, 93, 1, 2013, 23-33, 10.4141/CJAS2012-029]

The publisher's version is available at:

[http://www.nrcresearchpress.com/doi/pdf/10.4141/cjas2012-029]

When citing, please refer to the published version.

Link to this full text:

[http://hdl.handle.net/2318/127284] 


\title{
Radio frequency identification technologies for livestock management and meat supply chain traceability
}

\author{
P. Barge, P. Gay, V. Merlino, and C. Tortia ${ }^{1}$ \\ Dipartimento di Scienze Agrarie Forestali e Alimentari - Università degli Studi di Torino Via Leonardo da Vinci, \\ 44 - 10095, Grugliasco, Torino, Italy. Received 21 March 2012, accepted 18 September 2012.
}

\begin{abstract}
Barge, P., Gay, P., Merlino, V. and Tortia, C. 2013. Radio frequency identification technologies for livestock management and meat supply chain traceability. Can. J. Anim. Sci. 93: 23-33. Animal electronic identification could be exploited by farmers as an interesting opportunity to increase the efficiency of herd management and traceability. Although radio frequency identification (RFID) solutions for animal identification have already been envisaged, the integration of a RFID traceability system at farm level has to be carried out carefully, considering different aspects (farm type, number and species of animals, barn structure). The tag persistence on the animal after application, the tag-to-tag collisions in the case of many animals contemporarily present in the reading area of the same antenna and the barn layout play determinant roles in system reliability. The goal of this paper is to evaluate the RFID identification system performance and determine the best practice to apply these devices in livestock management. RFID systems were tested both in laboratory, on the farm and in slaughterhouses for the implementation of a traceability system with automatic animal data capture. For this purpose a complete system for animal identification and tracking, accomplishing regulatory compliance as well as supply chain management requirements, has been developed and is described in the paper. Results were encouraging for identification of calves both in farms and slaughterhouses, while in swine breeding, identification was critical for small piglets. In this case, the design of a RFID gate where tag-to-tag collisions are avoided should be envisaged.
\end{abstract}

Key words: Animal identification, farm and livestock management, radio frequency identification, traceability

Barge, P., Gay, P., Merlino, V. et Tortia, C. 2013. Technologies d'identification en radio fréquence pour la gestion du cheptel et la traçabilité de la chaine d'approvisionnement de la viande. Can. J. Anim. Sci. 93: 23-33. L'identification électronique des animaux peut être exploitée par les éleveurs comme une occasion pour améliorer l'efficacité de gestion du troupeau et la traçabilité. Bien que les systèmes RFID (identification par radio-fréquence) pour l'identification des animaux aient déjà été envisagés, l'intégration d'un système de suivi électronique au niveau de la ferme doit être effectué soigneusement en tenant compte de différents aspects (type d'exploitation agricole, le nombre et les espèces d'animaux, la structure de l'étable). La durabilité du transpondeur chez l'animal, la présence simultanée de plusieurs animaux dans la zone de lecture de l'antenne ainsi que l'agencement de l'étable jouent un rôle décisif sur le niveau de fiabilité du système. Le but de cet article est d'évaluer le rendement d'un système d'identification électronique RFID et de définir les meilleures pratiques pour son utilisation dans la gestion du troupeau. Le système d'identification électronique RFID a été testé en laboratoire, à la ferme et dans un abattoir commercial dans le but d'implanter un système de traçabilité permettant l'acquisition automatique des données des animaux. Dans le but d'améliorer l'acquisition automatique des données des animaux, la présente étude décrit le développement des solutions les mieux adaptées pour assurer la traçabilité tout au long de la chaine d'approvisionnement de la viande. Les résultats de l'identification des veaux tant à la ferme qu'à l'abattoir ont été encourageants alors que dans les élevages de porcs, l'identification des porcelets a été difficile. Dans ce dernier cas, il est essentiel de concevoir le portail RFID afin d'éviter les collisions entre les transpondeurs.

Mots clés: Gestion de la ferme d'élevage, identification électronique, RFID, traçabilité

The radio frequency identification (RFID) of each single head of cattle is already mandatory in many countries for some species (Smith et al. 2008). For example, to meet European Union requirement for meat import, Australia, in 1999, introduced a system for RFID identification and traceability of livestock (NLIS), which became compulsory in July 2005 for cattle; in the US, electronic identification is a voluntary program (NAIS), which has active since 2002, and some states, e.g., Michigan, officially started electronic animal-tracking

${ }^{1}$ Corresponding author (e-mail: cristina.tortia@unito.it).
Abbreviations: BDN, Banca dati dell'Anagrafe Zootecnica; DR, detection rate; DRE, dynamic reading efficiency; DS, detection share; ERP, enterprise resource planning; FDX, full duplex; HF, high frequency; HDX, half duplex; ICT, information and communication technology; ID, Identifier; GLM, generalized linear model; ISO, International Organization for Standardization; LF, low frequency; NAIS, National Animal Identification System; NLIS, National Livestock Identification System; PDA, personal digital assistant; RE, readability index; RF, radio frequency; RFID, radio frequency identification; $\mathbf{R R}$, reading rate; SDA, safe detection area; UHF, ultra high frequency; UNIANOVA, one-way analysis of variance; XML, extensible markup language 
in March 2007. In Canada, RFID identification for cattle has been mandatory since July 2010 .

In Europe, research was encouraged and supported by some European Union projects, e.g., the IDEA Project on animal identification (Ribó et al. 2001), and national and regional programs, and led to the development and testing of technological solutions for animal identification, by means of endoruminal, ear and injectable tags, and of reading devices [see for example: Caja et al. (1999), Lambooij et al. (1999), Klindtworth et al. (1999), Wismans et al. (1999), Smith et al. (2005)].

In the European Union, electronic identification of sheep and goats by radiofrequency has been mandatory since January 2010 (Reg. CE n. 21/2004, implemented by Commission Decision 2006/968/EC Commission followed by the amendments stated by the Commission Decision 2008/337/CE), while, for cattle, it is voluntary. For cattle, sheep and goats, the code structure and the operating frequencies must be compliant with the International Organization for Standardization 11784 and 11785 standards (International Organization for Standardization 1996a, b), which were defined and adopted in the early 1970s (Rossing 1999) and then approved by the International Committee for Animal Recording (ICAR 2005). No standards are at present defined for pig identification.

The ISO compliant RFID animal identification transponder operates at a frequency of $134 \mathrm{kHz}$ and stores a 64-bit code. Some limits of ISO standards, such as tag-to-tag collision, short reading distance and low reading rate, were reported by some researchers, who proposed solutions, such as the adoption of other frequencies, to cope with these problems (Leng et al. 2005; Leong et al. 2007; Gay et al. 2008). At present, only passive and read-only transponders are allowed for animal identification. The ISO solution of 64-bit coded tags limits the complexity of the transponders and their cost (Saa et al. 2005), but requires the permanent availability of a database to retrieve the necessary information. The introduction of new ISO standard, ISO 14223:2003 (International Organization for Standardization 2003) could allow the exploitation of the new possibilities offered by RFID technology (Sydänheimo et al. 2006). In particular, this ISO standard specifies the air interface between the transceiver and the advanced transponder used in the radio frequency identification of animals under the condition of full upward compatibility according to ISO 11784 and ISO 11785.

Some researchers proposed RFID solutions at higher frequencies $(\mathrm{HF}, 13.56 \mathrm{MHz})$ for pig identification at the feed trough, which allow the simultaneous detection of multiple transponder and use anti-collision systems (Reiners et al. 2009). Analogously, Leong et al. (2007) proposed the use of both HF and UHF (920-926 MHz) RFID tags for the identification of pigs in Australia.
The reliability of an RFID traceability system for livestock applications depends on two main factors: the persistence of the tag on the animal and the readability in different conditions in the stable. RFID system integration at the farm level should be carried out considering farm type, number and species of animals, as well as barn structure.

The goal of this paper, which reports the results of the research project InfoMeat, is to present technical solutions for the electronic identification of calves and pigs using RFID systems. The proposed solutions, which involve different types of RFID transponders, readers and software and the planning of equipment layouts in the stable, have been tested both in the laboratory and in barns.

\section{MATERIAL AND METHODS}

With the aim of comparing technical characteristics of different systems for electronic animal identification and determining best practice parameters for RFID systems integration in pig and calf barns, antennas and tags of different brands and dimensions were tested in static and dynamic conditions, first in the laboratory and then in barn trials. Single and multiple tag readings were considered. This last condition, which occurs when two or more animals are in the area read by the same antenna, typically presents tag-to-tag collisions that could drastically affect the detection performances and accuracy of the system. The breeding stable experimentation was conducted to evaluate the robustness and the reliability of the devices (tags and antennas), the tag persistence after application to the animal and the effectiveness of the designed layout solutions.

\section{Equipment}

Six different types of electronic button-type ear tags were tested. Table 1 reports brand, model, interrogation protocol and coil dimension.

Three fixed systems with panel antennas and one hand-held wand reader were compared. The fixed systems considered were:

- an Edit-ID (Auckland, New Zealand) system composed of a reader (model Race reader control unit) and a Crush panel antenna $(650 \times 850 \mathrm{~mm})$. The reader was connected to a PC for data storage by RS-232 serial port.

- a Gallagher BR 600 and a Gallagher BR 1300 (Hamilton, New Zealand) Smart Reader systems, which embed the same reader but differ for the antenna size $(600 \times 800 \mathrm{~mm}$ and $600 \times 1300 \mathrm{~mm}$, respectively). These systems work stand alone, as they can store data internally and are powered by an internal battery. Data download was performed via RS-232 serial port. 
Table 1. Brand, model, interrogation protocol and dimensions of the copper loop coil of the ear tags tested. Electronic tags applied to piglets $\left(A_{h}, B_{f}, C_{f}\right.$, $\left.D_{f}\right)$ and to calves $\left(E_{f}, F_{f}\right)$

\begin{tabular}{|c|c|c|c|c|c|c|}
\hline & \multirow[b]{2}{*}{ Brand } & \multirow[b]{2}{*}{ Model } & \multirow[b]{2}{*}{ Interrogation protocol } & \multicolumn{3}{|c|}{ Coil dimensions (mm) } \\
\hline & & & & External diameter & Internal diameter & Thickness \\
\hline $\mathrm{A}_{\mathrm{h}}^{\mathrm{z}}$ & Allflex & V3 LW & HDX & 22.5 & 12.5 & 0.6 \\
\hline$B_{f}$ & Allflex & LW & FDX-B & 22.5 & 19.5 & 2.0 \\
\hline $\mathrm{C}_{\mathrm{f}}$ & Caisley & Multiflex & FDX-B & 27.0 & 20.0 & 2.0 \\
\hline$D_{f}$ & Hauptner \& Herberholz & Neoflex & FDX-B & 21.0 & 17.0 & 2.0 \\
\hline$E_{f}$ & Zeetag & Large animal button & FDX-B & 28.0 & 24.0 & 1.5 \\
\hline $\mathrm{F}_{\mathrm{f}}$ & Demaplast & $0370 \mathrm{~F}$ & FDX-B & 28.0 & 20.0 & 0.9 \\
\hline
\end{tabular}

${ }^{\mathrm{z}}$ Subscript letters indicate the interrogation protocol: $\mathrm{h}=\mathrm{HDX}, \mathrm{f}=\mathrm{FDX}-\mathrm{B}$

The portable wand (total length $815 \mathrm{~mm}$, diameter $35 \mathrm{~mm}$ ) was composed of an antenna (500 mm length) and a handle that integrates a controller, a display and an on-board battery. Data download was guaranteed by a USB connection.

All these systems were ISO 11784/11785 compliant, operating at $134.2 \mathrm{kHz}$, and were enabled to read both half duplex (HDX) and full duplex (FDX-B) tags.

\section{Reading Range}

To evaluate the reading range of the different antennatag couplings, tested tags were suspended at half the height of the antenna, according the following tag vs. antenna coil plane orientations: parallel, perpendicular and, finally, with the tag coil plane oriented towards the centre of the antenna.

Tags were moved towards the antenna until a stable detection was established. In this way a section of the read volume at half height of the antenna was obtained and quoted measuring the distances occurring from these points to the panel.

The reading range of the wand antenna was determined by orienting the tag radially towards a point $80 \mathrm{~mm}$ below the antenna edge.

\section{Dynamic Reading Performance}

For the evaluation of dynamic reading performance with fixed antennas, a wooden trolley, pulled by a rubber belt activated by an electric DC motor, was purpose built to simulate the passage of the animals. On this trolley, which was pulled on rails at a constant speed in front of the antenna, different combinations of tags at the height of the centre of the antenna were mounted. During the trials, the trolley speed was arranged from 0.8 to $3 \mathrm{~m} \mathrm{~s}^{-1}$. The number of readings of each tag was counted during the passage in front of the antenna.

\section{Reading Rate}

The reading rate (RR) of a moving single transponder was calculated as the number of correct readings in a time unit, averaged on at least 10 trials. In this case, to prevent possible cross-interference with other transponders, only one tag at a time was mounted on the trolley.

\section{Collisions}

To evaluate the effect of multiple animals passing in the reading area of the antenna, two or four tags were installed on the trolley at increasing distances. Experiments mounting various combinations of tags (HDX and FDX of different producers) were conducted. This allowed determination of reading errors due to tag-totag collisions in the different situations. The RR of each single tag in a multiple tag configuration was registered and the detection share (DS), was calculated as the ratio of the considered tag readings to the number of total readings of the tags present.

The tags were spaced on the trolley at increasing distances to simulate the conditions on small animals, for example small piglets, where the distances among the tags could, in practice, be very small.

The real effect of tag-to-tag collisions in livestock radio frequency identification was evaluated in the calf and swine breeding farms involved in the project.

\section{In-farm Testing}

To integrate the RFID system at the farm level, representative breeding farms in Piedmont were chosen considering breeding management, number and type of animals as well as barn structure.

The experimentation involved four different cattle and two swine farms. Farm 1 and Farm 2 were traditional family-run and closed cycle Piedmont breed cattle farms (80 and 200 head, respectively). Farm 3 and Farm 4 were intensive livestock breeding farms, with more than 1000 head. In this case, Piedmont breed and other French breeds calves (Charolaise, Blonde d'Aquitaine) were purchased in France or in Piedmont and fattened in the farm (open cycle).

Homogeneous groups of calves were tagged at different ages. Calves were identified applying a RFID FDX-B ear tag transponder (the bigger ones, type $E_{f}$ or $\mathrm{F}_{\mathrm{f}}$ ) on the left ear with the appropriate pliers. The electronic code was registered in the BDN.

The average time required for tagging was recorded, as well as reading efficiency and losses or breakage of the transponders. To this extent, the performances in static and dynamic conditions were quantified adopting the readability index $(\mathrm{RE} \%=$ tags which have been 
$\mathrm{read} /$ tags applied $\times 100$ ) and the dynamic reading efficiency index $(\mathrm{DRE} \%=$ tags which have been read/ readable tags present $\times 100$ ) as proposed by Caja et al. (1999) using panel antennas fixed in a gate on the same side of the identified ear.

The in-farm research activity, which lasted $2 \mathrm{yr}$, was conducted on a lot of 160 identified piglets/year for both farms (Farm 5 and Farm 6), applying an electronic tag on the right ear, as the left ear was already marked by a tattoo. Four sub-lots of 40 animals each were formed, homogeneous for applied transponder type $\left(A_{h}, B_{f}, C_{f}\right.$ or $\mathrm{D}_{\mathrm{f}}$ ). In Farm 5, the piglets were tagged at weaning (day 28 from birth), while in Farm 6, transponders were applied on day 59 from birth. Two operators, one holding the piglet and the other applying the identifier, performed tagging; the average time required per piglet was estimated measuring the time required to apply the tag at the entire lot.

In Farm 5, lots of 40 piglets were kept indoors, each in a $12-\mathrm{m}^{2}$ pen, with a plastic fully slatted floor, fed ad libitum and with free access to bowl water drinkers. Disadvantaged or dead pigs were removed. In Farm 6, weaned piglets were bred together in a herd of 400 animals in an an open structure with indoor as well as outdoor space.

The dynamic readings were performed on day 28 (only for the group in Farm 5), 59, 76, 93 from birth, guiding group of 40 pigs through a gate of different width equipped with a static reader (Edit-ID and Gallagher SmartReader RD 600). Each dynamic reading experiment, consisting of the transit of the sub-lot of pigs through the gate, was repeated three times to determine reading performances in terms of DRE\%. Finally, animals were identified the day of transport to slaughter when entering the truck.

\section{In-slaughterhouse Testing}

In the corridor of the slaughterhouse, the electronic codes contained in the ear tags were read using a panel antenna (Edit-ID Crush) just before the animals were stunned. The antenna was fastened to the horizontal iron bar of the slaughterhouse admission corridor at the same height as the animals' heads. A web-based system for meat traceability paired to RFID data capture was adopted (Barge et al. 2009).

In slaughterhouses, RFID systems determine the exact sequence in the queue of animals entering the slaughtering plant. At this time, the availability of all the data about the animal is essential for slaughterhouses as they are required to transmit the information for quality certification and for mandatory slaughter documents. The system was based on the slaughterhouse client on which resides an ActiveX control developed to interface the RFID antenna and to automatically insert the electronic ID code in a database retrieving all the necessary data from the national register (BDN) requested to fulfil the subsequent traceability mandatory data interchange. The information retrieved from the national database is automatically registered in the slaughterhouse enterprise resource plan, which produces the requested slaughtering documents and shares information through a collaborative network among other stakeholders of the supply chain that can add further data (e.g., mandatory, voluntary, processing parameters, costs). This information can then be accessed down the supply chain, at the prescribed detail level, opening the system at new business-to-business data interchanges.

\section{Statistical Analysis and Experimental Design}

To evaluate the effects of different factors (antenna type, tag type, distance among tags and position in the tag queue scheme) on the reading rate of the different tag combinations, a generalized linear model (GLM) with interactions was adopted. A one-way analysis of variance (UNIANOVA) procedure for regression analysis and analysis of variance of the dependent variable by the considered factors and interactions was used. The antenna types compared were the Edit-ID and Gallagher Smartreader BR 600. Six repetitions for each factor combination were acquired. The factor position in the tag queue was considered only in the case of four tags. In the case of four tags of the same type the experimentation regarded only the Edit-ID antenna. Means were then compared by a post-hoc Tukey test. SPSS $^{\circledR}$ Statistics 17.0 statistical package was used to process data.

For evaluating the effect of tag type when two tags were paired and in the case of four tags of the same type, only on the Edit-ID antenna was used.

\section{RESULTS Laboratory Experimental Trials}

\section{Antenna Detection Area}

The shape of the transponders' detection zone according to the orientations considered was similar for the three tested panel antennas. In the case of the tag oriented towards the antenna centre (radial orientation), the detection zone appears to be formed by one lobe, while in the case of the tag and antenna coil in parallel planes, it is composed of one main lobe bordered by two secondary lobes (Fig. 1). In the case of the tag oriented with its coil plane perpendicular to the antenna, the detection zone was composed of two symmetric lobes in front of the antenna's left and right edges, while in the area just in front of the antenna panel a shadow no-reading zone was registered.

For all tag orientations and antennas, the resulting detection zone, whose dimension varied as a function of the tag size and the antenna type, always exceeded the panel dimensions.

Six parameters were defined to characterize and compare the dimensions of the detection zone for the different tag-antenna couplings, as represented in Fig. 1. 


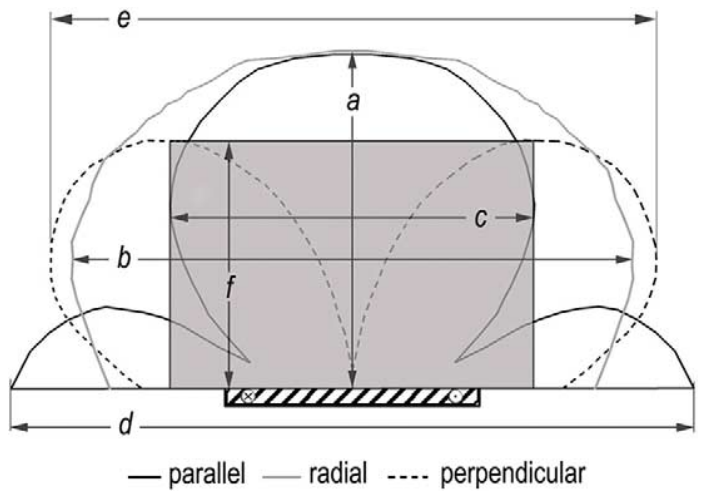

Fig. 1. Intersection of the antenna detection areas obtained according to the different tag-antenna orientations (tag coil plane parallel and perpendicular to the antenna plane or radially oriented towards the antenna centre). The Safe Detection Area (SDA), inside which the tag is more likely to be detected, is shaded.

The measured values for these parameters are reported in Table 2.

Combining the shapes of the detection area relative to the different tag-antenna orientation, we identified a rectangular region $(f \times c)$, which we refer to as the "safe detection area" (SDA), inside which the tag is likely to be read, with a high degree of confidence. Even if the transponder is identified in all the examined orientations, only in the intersection of the three detection areas the tag succeeds to be identified when it moves through the SDA. The detection could also occur outside the margins of the antenna. The dimensions of the detection area are related to panel shape as well as tag coil size.

In Table 2, the SDA length $(c)$ and width $(f)$ for different tag-antenna combinations are reported.

Table 2. Length of the dimension parameters for different tag/antenna combinations. Safe detection area is a rectangle of length $c$ and width $f$

\begin{tabular}{|c|c|c|c|c|c|c|}
\hline \multirow[b]{2}{*}{ Tag type } & \multicolumn{6}{|c|}{ Dimension parameters (mm) } \\
\hline & $a$ & $b$ & $c$ & $d$ & $e$ & $f$ \\
\hline \multicolumn{7}{|c|}{ Gallagher BR 600} \\
\hline$A_{h}$ & 710 & 1060 & 800 & 1400 & 1210 & 550 \\
\hline$B_{f}$ & 830 & 1240 & 800 & 1500 & 1320 & 630 \\
\hline$C_{f}$ & 930 & 1400 & 900 & 1600 & 1490 & 650 \\
\hline$D_{f}$ & 810 & 1260 & 700 & 1500 & 1330 & 580 \\
\hline \multicolumn{7}{|c|}{ Edit-ID Crush Antenna } \\
\hline$A_{h}$ & 690 & 1230 & 800 & 1500 & 1420 & 520 \\
\hline$B_{f}$ & 760 & 1270 & 820 & 1600 & 1510 & 580 \\
\hline $\mathrm{C}_{\mathrm{f}}$ & 900 & 1480 & 960 & 1800 & 1600 & 660 \\
\hline$D_{f}$ & 750 & 1190 & 800 & 1580 & 1450 & 540 \\
\hline $\mathrm{E}_{\mathrm{f}}$ & 860 & 1370 & 860 & 1550 & 1470 & 640 \\
\hline \multicolumn{7}{|c|}{ Gallagher BR 1300} \\
\hline$A_{h}$ & 790 & 1290 & 960 & 1700 & 1460 & 650 \\
\hline$B_{f}$ & 1020 & 1540 & 1380 & 1900 & 1650 & 750 \\
\hline $\mathrm{C}_{\mathrm{f}}$ & 1150 & 1740 & 1500 & 2100 & 1820 & 840 \\
\hline$D_{f}$ & 960 & 1460 & 1240 & 1800 & 1550 & 700 \\
\hline
\end{tabular}

The Gallagher BR 600 antenna reading area is more deep than wide; the measured parameters indicate a comparable detection area, but with longer depth parameter ( $a$ and $f$ ) than for the Edit-ID Crush antenna.

The Gallagher BR 1300 detection zone parameters were higher due to the bigger panel size.

The detection area of the hand-held wand antenna is reported in Fig. 2. The shape of the area is formed by two lobes. The detection area is symmetric around the wand axis. The maximum reading distance was $30 \mathrm{~cm}$ with tag $E_{f}$.

\section{Dynamic reading performances}

Reading rates of each single tag (RR) ranged from 3 to 5 synchs per second. The identification cycle of the HDX transponder $\left(\mathrm{A}_{\mathrm{h}}\right)$ was faster (average $\mathrm{RR}=5.10 \pm 0.35$ readings $\mathrm{s}^{-1}$ ), than for FDX tags (average $\mathrm{RR}=3.42 \pm$ 0.54 readings $\mathrm{s}^{-1}$ for $\mathrm{B}_{\mathrm{f}}, 4.14 \pm 0.35$ readings $\mathrm{s}^{-1}$ for $\mathrm{C}_{\mathrm{f}}$, $3.30 \pm 0.21$ readings $s^{-1}$ for $\mathrm{D}_{\mathrm{f}}$ and $3.66 \pm 0.29$ readings $\mathrm{s}^{-1}$ for $\mathrm{E}_{\mathrm{f}}$ ). Series of trials were made to assess collisions when multiple tags were passing contemporarily in the detection area. When two tags were paired, RR was affected by cross-interference among tags. Table 3 reports the RR of each transponder during dynamic reading in free space, when two different tags were mounted on the trolley in all possible combinations (i.e., $A_{h}-B_{f}, A_{h}-C_{f}, A_{h}-D_{f}, B_{f}-C_{f}, B_{f}-D_{f}$ or $C_{f}-D_{f}$ ), at different distances, in the range $0-100 \mathrm{~cm}$.

When two tags were close to each other, RR was, for both tags, lower than in the case of one tag alone. The effect of the factor tag was significant for $P<0.001$ in all the pair-wise comparison. The effect of the factor distance among tags was significant for $P<0.01$ in all cases, except for the $A_{h}$ and $B_{f}$ pair of tags. This was the case when two tags of the same coil size but of different communication protocol were paired; RR was not significantly different for the two tags even when

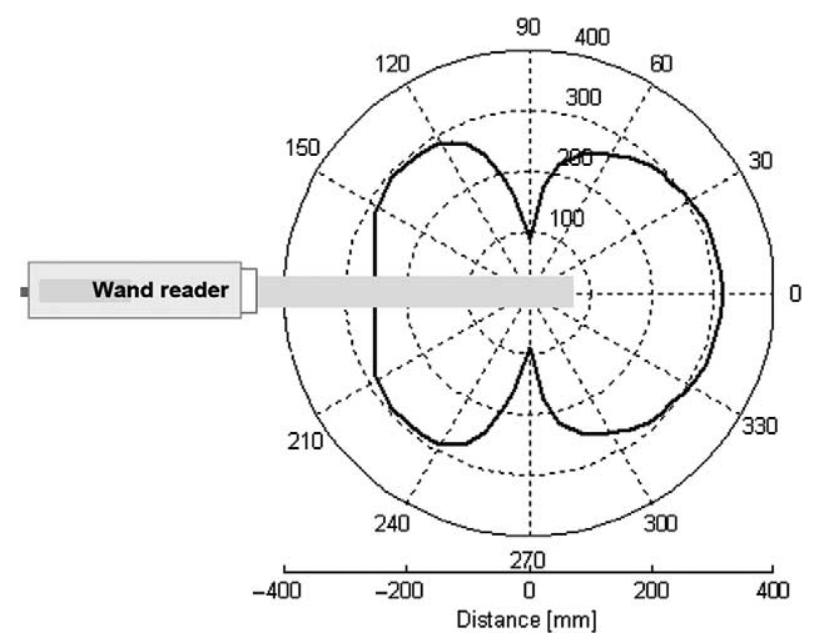

Fig. 2. Wand hand-held antenna detection area measured using $\operatorname{tag} \mathrm{E}_{\mathrm{f}}$. 
Table 3. Mean reading rate $(R R)$ of each transponder during dynamic reading in free space, tag mounted in pairs $\left(A_{h}-B_{f}, A_{h}-C_{f}, A_{h}-D_{f}, B_{f}-C_{f}\right.$, $B_{f}-D_{f}$ or $\left.C_{f}-D_{f}\right)$ at different distances $(0-100 \mathrm{~cm})$

\begin{tabular}{lllllll}
\hline & \multicolumn{2}{c}{$\mathrm{A}_{\mathrm{h}}$} & \multicolumn{2}{c}{$\mathrm{B}_{\mathrm{f}}$} & \multicolumn{2}{c}{$\mathrm{C}_{\mathrm{f}}$} \\
\hline $\mathrm{Bf}$ & 2.26 & 3.16 & & & & \\
$\mathrm{C}_{\mathrm{f}}$ & $2.64^{*}$ & $2.84^{*}$ & $2.40^{*}$ & $1.72^{*}$ & & \\
$\mathrm{D}_{\mathrm{f}}$ & $2.06^{*}$ & $2.90^{*}$ & $1.60^{*}$ & $1.78^{*}$ & $1.48^{*}$ & $2.50^{*}$ \\
\hline
\end{tabular}

*Samples are different for factor distance among tags at $P<0.01$.

they were placed in contact. In fact, ISO compliant antennas continuously switch from HDX to FDX-B, allowing each of the two tags to communicate separately, undisturbed. For the same reason, the pairing of FDX-B tags with tag $A_{h}$ has a minor effect on reading rate with respect to the pairing with other FDX-B tags.

When two FDX-B transponders were in the detection area, in contact, the RR was totally in favour of one tag only (always significant at Tukey's post-hoc test, $P<0.05)$. The bigger transponder always achieved a faster RR.

When four different types of tags were mounted on the trolley at increasing distances, the effect of the factors antenna, tag, distance among the tags and position in the tag queuing scheme resulted in highly significant effects on transponder RR $(P<0.001)$, as well as for the factor position and distance when the four tags of the same brand and model were mounted on the trolley $(P<0.001)$.

The effect of all the factors' interactions was highly significant for both models $(P<0.001)$.

The overall mean RR was higher for the EditID antenna (1.25 readings $\left.\mathrm{s}^{-1}\right)$ compared with the Gallagher $\left(0.72\right.$ readings $\left.\mathrm{s}^{-1}\right)$.

In the case of four different tags, the higher performances were ascribed to the HDX tag $A_{h}$ (Table 4). These results agree with the previous RR results in tag pairwise comparison as well as for the RR of the tag alone.

The effect due to collision with transponders of the same brand significantly reduced the performance in terms of RR in the case of four tags of the same brand

Table 4. Effect of the factors tag type $\left(A_{h}, B_{f}, C_{f}\right.$ and $\left.D_{f}\right)$, position in the queuing sequence (I, II, III, IV) and distance among tags (2.5, 5, 10, 20 and $30 \mathrm{~cm}$ ) on the reading rate (RR)

\begin{tabular}{lccccc}
\hline Factor tag & $\mathrm{D}_{\mathrm{f}}$ & $\mathrm{B}_{\mathrm{f}}$ & $\mathrm{C}_{\mathrm{f}}$ & $\mathrm{A}_{\mathrm{h}}$ & \\
\hline \multirow{3}{*}{ Factor position } & $0.34 a$ & $0.83 b$ & $0.99 c$ & $1.31 d$ & \\
& III & II & IV & I & \\
Factor distance & $0.66 e$ & $0.69 e$ & $0.98 f$ & $1.14 g$ & \\
& 2.5 & 5 & 10 & 20 & 30 \\
& $0.30 h$ & $0.71 i$ & $0.86 l$ & $1.10 m$ & $1.37 n$
\end{tabular}

$a-d, e-g, i-n$ Letters denote groups significantly different by the Tukey test. model for all tag types, which ranged from a mean of 0.35 to 0.61 readings $\mathrm{s}^{-1}$ for each tag, with a stronger effect on the HDX transponder as the mean RR of tag $A_{h}$ resulted in the lower value. This is also true because of the significance of the interaction of the factor tag and position, which is also high for this model.

In all the experiments, tags in the first and last positions gave high DS and RR, while those in the middle (second and third positions) were significantly less favoured in the case of different tags as well as tags of the same model. In both models, mean reading rates of the second and third positions were grouped into two statistically homogeneous sets (at Tukey's test, $P<0.05$ as may be seen in Tables 4 and 5). This could be due to the fact that the first and the last transponders, even for a short time period, remain alone in the detection area, while the tags in the second and third positions are always in collision with at least another transponder. Considering the differences in the transponder sequence, it has been noted that, for both the tested antennas, if the $A_{h}$ transponder was in the second or third position, the DS of each tag was more balanced. In contrast, when the HDX transponder was placed in the more favourable position, very low DS of one transponder type was recorded, if placed in the middle of the sequence.

The effect of position in the queue had different effects when four transponders of the same brand and model were mounted on the trolley (Table 5). In this case, RR of the HDX tags was very low and the DS was totally in favour of the first tag in the queue. As in the case of four different tags, the second and third positions were the less favoured, and in the case of the $\mathrm{A}_{\mathrm{h}}$ tags were practically not detected in the distance range considered.

For the factor distance, RR increased at increasing distances among the tags in the considered range. In the case of four different tags, five significantly different groups were distinguished by Tukey's test $(P<0.05)$, while in the case of four identical transponders, only four groups were individuated, being the

Table 5. Effect of the factor position (I to IV) on the mean RR (number of correct readings $s^{-1}$ ) of each transponder when four transponder of the same model were mounted on the trolley at increasing distance (range 0-100 cm)

\begin{tabular}{lcccc}
\hline & \multicolumn{4}{c}{ Position in the queue } \\
\cline { 2 - 5 } Tag & I & II & III & IV \\
\hline $\mathrm{A}_{\mathrm{h}}$ & $0.84 c$ & $0.06 a$ & $0.00 a$ & $0.53 b$ \\
$\mathrm{~B}_{\mathrm{f}}$ & $0.83 e$ & $0.15 d$ & $0.27 d$ & $1.00 f$ \\
$\mathrm{C}_{\mathrm{f}}$ & $0.98 g$ & $0.15 h$ & $0.23 h$ & $1.07 i$ \\
$\mathrm{D}_{\mathrm{f}}$ & $1.01 l$ & $0.15 m$ & $0.16 m$ & $0.88 n$ \\
\hline
\end{tabular}

$a-c, d-f, g-i, l-n$ Letters denote groups significantly different by the Tukey test. 
smaller distance values $(2.5$ and $5 \mathrm{~cm})$ not significantly different.

The second and third transponders were not detected at all if the mutual distance was less than or equal to $30 \mathrm{~cm}$ in the case of $\mathrm{A}_{\mathrm{h}}$ HDX transponder. In the case of four FDX-B tags of the same model and brand, the second and third tags were detected for distances greater than $20 \mathrm{~cm}$. This was confirmed for all the types of FDX tags considered.

The effect of the antenna is very low in the interaction between antenna and tag factor for tag $\mathrm{D}_{\mathrm{f}}$, which means $\mathrm{RR}$ is equal for both antennas, while, especially for tag $\mathrm{A}_{\mathrm{h}}$ and $\mathrm{B}_{\mathrm{f}}$, interaction among the Edit-ID antenna gave good results in terms of RR.

The collision phenomenon is very similar for the EditID and Gallagher antennas, but the influence of the antenna is greater for the first and last positions (I and IV) in the case of the Edit-ID antenna.

The enhancement of RR due to the Edit-ID antenna is significantly more pronounced at greater distances among the tags, if compared with the results obtained with the Gallagher antenna.

\section{Farm Experimental Trials}

\section{Dynamic Reading Performances of Transponders Applied to Piglets}

The average requested time for transponder application was $17 \mathrm{~s}$ per piglet at day 28 , while the same operation was performed in $22 \mathrm{~s}$ per piglets at day 59. The dynamic reading, performed just after transponder application, was very difficult as small piglets were allowed to pass in groups in front of the antenna, generating tag-to-tag collisions in the communication with the antenna. A gate of $300 \mathrm{~mm}$ was too wide for accurate animal identification at this stage (Table 6).

In fact, from the data recorded in the laboratory, the readings could occur for a length of $1500 \mathrm{~mm}$ (parameter $d$ in Fig. 1), while the average length of a piglet at this age is $650 \div 700 \mathrm{~mm}$. This means that at least two piglets could be in the reading area at the same time, even if the piglets pass one at a time.

Table 6. Dynamic reading efficiency (\%) by Edit-ID panel antenna of different types of transponder $\left(A_{h}, B_{f}, C_{f}\right.$ and $\left.D_{f}\right)$ applied on piglets and read at different ages $(28,59,77$ and $92 \mathrm{~d})$ in two passage width (200 and $300 \mathrm{~mm})$

\begin{tabular}{|c|c|c|c|c|c|}
\hline & \multirow[b]{2}{*}{ Transponder type } & \multicolumn{4}{|c|}{ Age of piglets (d) } \\
\hline & & 28 & 59 & 77 & 92 \\
\hline \multirow[t]{4}{*}{ Passage width $200 \mathrm{~mm}$} & $A_{h}$ & 90 & 95 & 100 & 97 \\
\hline & $B_{f}$ & 100 & 97 & 97 & 94 \\
\hline & $C_{f}$ & 93 & 100 & 100 & 98 \\
\hline & $D_{f}$ & 92 & 98 & 99 & 97 \\
\hline \multirow{4}{*}{ Passage width $300 \mathrm{~mm}$} & $A_{h}$ & 63 & 87 & 94 & 100 \\
\hline & $B_{f}$ & 91 & 86 & 94 & 97 \\
\hline & $C_{f}$ & 80 & 87 & 97 & 96 \\
\hline & $\mathrm{D}_{\mathrm{f}}$ & 53 & 83 & 100 & 100 \\
\hline
\end{tabular}

At this age of the animals ( $28 \mathrm{~d})$, readability did not increase at acceptable levels for most of the transponder types even if the passage was reduced to $200 \mathrm{~mm}$ (Table 6). Moreover, in this narrow passage, piglet flux in front of the antenna was too slow for normal breeding practice. An even wider gate of $450 \mathrm{~mm}$, used more in farm practice, gave very poor reading results, in particular for piglets at days 29 (data not reported). At the end of the fattening period, when it was possible to convey the swine in a single head queue, avoiding the simultaneous passage in front of the antenna of more than one animal, the reliability of the system was improved. With piglets of $92 \mathrm{~d}$ of age, the reading was more precise at both widths, but it was very often not complete. The identification of the swine at this age is very important because the animals are normally moved to a fattening pen. Dynamic reading by a gate with a passage width of $200 \mathrm{~mm}$ gave good results from the 77 th day from birth, for all the tags.

From these results, in critical conditions $C_{f}$ and $B_{f}$ transponders gave the best results, while the $\operatorname{HDX}\left(\mathrm{A}_{\mathrm{h}}\right)$ transponder did not give better results than FDX-B tags $\left(\mathrm{B}_{\mathrm{f}}, \mathrm{C}_{\mathrm{f}}, \mathrm{D}_{\mathrm{f}}\right)$, in dynamic reading performance. These results agree with laboratory test trials.

The dynamic reading efficiencies of two different antennas were compared in the identification of 2mo-old piglets in a gate wide enough to allow the passage of only one piglet at a time. From the results of this trial, both the antennas gave good results, with 99\% DRE for Edit-ID reader and 98\% DRE for Gallagher antenna (data not reported).

A good queuing of piglets with an apposite structure, which progressively restricts the passage, resulted in benefits to the accuracy of the readings.

A mortality rate of $10 \%$ was recorded during the $2 \mathrm{yr}$ of research activity, mainly due to PRRS virus disease.

Transponder persistence was evaluated by means of a hand-held wand antenna checking every head of swine at $84,148,217$ and $245 \mathrm{~d}$ from birth. Tag persistence ranged from $100 \%$ at $56 \mathrm{~d}$ to $88.4 \%$ at $217 \mathrm{~d}$ from tagging. Losses of different tag types at $217 \mathrm{~d}$ are reported in detail and were 4.0 for $A_{h}, 6.5$ for $C_{f}, 6.6$ for $\mathrm{D}_{\mathrm{f}}$ and 15.6 for $\mathrm{B}_{\mathrm{f}}$. The FDX-B tags $\mathrm{B}_{\mathrm{f}}$ showed the major percentage of losses. In Farm 6, losses were higher, but were lower than data reported by Caja et al. (2005), which indicate losses ranging from 12 to $72 \%$.

Losses were mainly due to the tag tear from the auricle and the detachment of the two parts constituting the tag.

\section{Dynamic Reading Performances of Transponders Applied to Beef Cattle}

Calves were easily tagged in Farm 4 because of the presence of suitable facilities (narrow corridors for animal medical treatments), which allow animal handling and restraint. Readability (DRE\%) through panel antenna (Edit-ID and Gallagher BR600) resulted 100\% 
in dynamic tests. In Farm 2 and Farm 3 the tagging operation was quite difficult because of the loose housing breeding system and the age of the calves. In Farm 1, to perform the tagging, the animal was captured and the tagging time ranged from 1 to 2 min per head. Identification of newborn calves, which is the best age to apply the ear tag, was very easy. In 5 mo, readability by wand of $E_{f}$ tags was always $100 \%$ in all the farms considered (Table 7) as well as in slaughterhouses, but in some cases the only method of reading by wand was for the farmer to enter the box (e.g., in Farms 1 and 3), which was dangerous due to the possibility of being kicked by the animal. The RE\% by the wand was $60-70 \%$ for $F_{f}$ tags. This was due to the fact that in this transponder the microchip is not included in a plastic material like in the other models, and was damaged by vibrations due to animal movements. The damage rate of $F_{f}$ ear tags increased with time after application and, when animals were slaughtered (at day 139 in Farm 3 and day 166 in Farm 4), only a few of them were functioning correctly (70 and $50 \%$, respectively). This was because in the $\mathrm{F}_{\mathrm{f}}$ tag the welding of the internal microchip was broken because it was not enclosed well in the plastic of the ear tag.

Tag persistence was 100\% in Farms 4 and 2 and 98\% in Farms 1 and 3. The particular configuration of the stunning cage at the slaughterhouse makes the identification very reliable, achieving $100 \%$ of DRE $\%$ in the experiment. The developed software allowed the realtime retrieval of the correct information from the BDN that was used to automatically fill the slaughterhouse database forms during stunning.

\section{DISCUSSION}

When a RFID system has to be integrated into a traceability system for animal identification, animal shape has to be related to the reading area of the antenna and probability of collision among tags has to be considered.

The SDA of the adopted tag-antenna combination should be determined precisely before planning the layout of a RFID gate to be installed in the barns, e.g., to determine the dimensions and shape of the animal passage where the antenna should be mounted. In fact, in order to be reasonably sure to detect the transponder even in the worst case (null orientation), the distance between the transponder and the antenna surface should not be greater than the $c$ parameter length.

The $f$ parameter indicates the width in front of the antenna of the zone where the tag is detected in at least one of the considered orientations. However, particularly when dealing with small animals like piglets, the overall detection area, i.e., the area obtained considering all the possible orientations, should be taken into account. In this case, many identifiers could, in fact, be present contemporarily in the detection area, leading to tag-to-tag collisions that can cause wrong or missed detection and/or faults in establishing the right queue. For this reason, parameters $d$ and $e$ become important for the design of gate layouts and should be taken into account with respect to animal length.

The availability of information about the reading areas and dynamic reading performances are fundamental to integrate RFID systems in real traceability applications.

The collision among tags resulted in a strong decrease of the reading rate when more than one tag was present in the detection area and, from the obtained results, the worst case was that of the presence of multiple tags of the same model and brand, especially for the HDX tag considered.

As the breeder usually buys tags of the same model, the choice of tag must take into account that the use of HDX transponders, even if number of readings is higher, enhances tag-to-tag collision problems. In addition, the cost of HDX tags is usually higher.

From results obtained on the persistence of the transponder and readability, RFID identification by means of ear tags is suitable for calves destined for slaughter as in their short life the loss of transponder is limited, as reported in literature (Ghirardi et al. 2004; Caja et al. 2005). However, the peculiarity of each breeding system must be carefully evaluated for RFID system integration for farm management. In fact, due to the very short reading distance, the identification of bovines restricted into barn boxes is not applicable at $134 \mathrm{kHz}$, either with fixed or portable antennas. In this latter case, approaching the animal could even be dangerous for the operator. For this reason, barn

\begin{tabular}{|c|c|c|c|c|c|c|c|c|}
\hline & Year & Breed $^{\mathbf{z}}$ & Tag type & Identifiedanimals $\mathrm{N}$ & Age (mo) & Persistence $(\%)$ & RE wand (\%) & DRE $(\%)$ \\
\hline \multirow[t]{2}{*}{ Farm 1} & 2007 & \multirow[b]{2}{*}{ PI } & $\mathrm{E}_{\mathrm{f}}$ & 44 & $2-11$ & 98.0 & 100.0 & \multirow[t]{2}{*}{100.0} \\
\hline & 2008 & & $F_{f}$ & 43 & $0-8$ & 98.0 & 60.5 & \\
\hline \multirow[t]{2}{*}{ Farm 2} & & PI & $\mathrm{E}_{\mathrm{f}}$ & 21 & $6-12$ & 100.0 & 100.0 & \multirow[b]{2}{*}{100.0} \\
\hline & 2007 & BA & $E_{f}$ & 22 & $9-10$ & 100.0 & 100.0 & \\
\hline Farm 3 & 2008 & PI & $F_{f}$ & 77 & $4-12$ & 98.5 & 60.0 & 100.0 \\
\hline Farm 4 & 2008 & $\mathrm{PI}, \mathrm{BA}, \mathrm{CH}$ & $F_{f}$ & 43 & $4-12$ & $100 \%$ & 50.0 & 100.0 \\
\hline
\end{tabular}

${ }^{\mathbf{z}}$ Breed notation: PI, Piedmont; BA, Blonde d'Aquitaine; $\mathrm{CH}$, Charolaise. 
structures where animals can be conveyed in corridors are suitable for traceability RFID systems integration.

As also noticed by Burose et al. (2010), in swine breeding, even though the adoption of an ear tag transponder was very promising, as the persistence of the ear tag was acceptable during the first 3 mo of the piglet's life, results are not yet satisfactory. The dynamic reading, in fact, gave good results only if carried on heads at $80 \mathrm{~d}$ from birth, while on small piglets dynamic reading rates were very poor. Before this age, the portable reader should be used as well as at the moment of tag application.

Due to the piglet's small dimensions, a smaller detection area reduced the problem of tag collision, as fewer piglets are allowed to remain in the detection field at the same time. To solve the problem, the use of smaller sized antennas and the synchronization of more than one panel are envisaged.

In previous work on piglet identification, some authors have also envisaged the use of HF RFID systems (Thurner and Wendl 2007; Hessel et al. 2008), which perform better when it is necessary to process data by anti-collision algorithms.

For cattle, RFID systems could be applied on the breeding farms as well as in slaughterhouses. On the farm, these systems could be used at different levels to record information about animals entering or being sold from the farm, sanitary treatments, and feeding (Trevarthen and Michael 2007). Mandatory information is stored for automatic animal registration and realtime data interchange with the BDN database. This is transferred to slaughterhouses and can be completed with additional data regarding voluntary meat labelling information, which is stored and certified by producer associations in the Infomeat database.

Farm management and mandatory registers are updated synchronizing mobile and fixed antennas with a proprietary database. With this objective, an ActiveX control is implemented on the client side to connect the RFID code with the requested database.

At the slaughterhouse, communication between remote hosts has been based on eXtensible Markup Language/Simple Object Access Protocol framework. XML grammar should be strictly derived from the Italian BDN XML in order to maintain a consolidated standard. A common standard for different worldwide data sharing is necessary as well as data communication among the different national registers. For example for the supply chain where calves are born in France and fattened in Italy a synchronization of the two national databases could enhance safety in data interchange.

Similar to the system proposed by Voulodimos et al. (2010) for farm management and Mutenje et al. (2012) for cattle handling, the ICT framework presented in this paper allows increased efficiency of the collection and management of traceability data, increasing reliability and reduced costs. This framework, improving, via realtime data exchange, safety and traceability (Dabbene and Gay 2011) and allowing a deep optimization of the supply chain (Schwägele 2005; Dabbene et al. 2008a, b), will enhance the competitiveness of the producers on the global market, favouring new forms of business-tobusiness electronic transactions (Liu and Shao 2012; Mingxiu et al. 2012).

Finally, the availability of a web-shared information system, for example the Infomeat platform, will allow the adoption of a common traceability path involving small producers and slaughterhouses, which can maintain traditional data recording even if the animal is identified with both an electronic and a visual device (Gay et al. 2007). All the other supply chain stakeholders can therefore, use the RFID.

\section{CONCLUSIONS}

The proper design of stable facilities for the automatic identification of head requires the characterization of the antenna-tag systems, considering the cases of identification both in static conditions, such as cattle in a box, and in dynamic conditions, for example what occurs for pigs and cows moving in corridors.

The results of the experimentation conducted on five farms, for cattle and swine, provided useful information about dynamic reading performances, tag-to-tag collisions and tag persistence and failure. For bovines, the proposed solutions, comprehensive of the RF equipment as well as appropriate layout of the farm building and facilities, are ready for application in breeding farms and slaughterhouses. In many cases, electronic identification can be used to automatically process data access to national registry databases. This process has been successfully tested at slaughterhouse where, after cattle identification, the developed ICT system automatically connected to the national registry database allowing real-time data synchronization. In swine breeding, the $\mathrm{RF}$ system should be ameliorated improving LF systems or using higher frequencies (HF or UHF) that could help to solve the problem of the detection of animal flow and, in the future, to lower the transponder cost.

The whole supply chain could take advantage of electronic identification and real-time availability of data on a collaborative network of shared platforms, supplied by the different stakeholders, with appropriate access permission levels.

\section{ACKNOWLEDGMENTS}

The research described in this paper was funded by Regione Piemonte and was supported by Producers Association COALVI, Asprocarne Piemonte and APS (Associazione Produttori Suini). The Authors wish to thank G. Tomatis, R. Ciravegna, L. Bersano, A. Persico and C. Pelassa, Consorzio Grossisti Industria Commercio Carni Coop. a.r.l., for their active and helpful cooperation in farms or slaughterhouses. 
Barge, P., Campo, M. W., Piccarolo, P., Racioppi, F., Torassa, C. and Tortia, C. 2009. Web based systems and RFID for meat traceability. Proceedings of XXXIII CIOSTA - CIGR V Conference "Technology and management to ensure sustainable agriculture, agro-systems, forestry and safety", Jun. 17-19, Reggio Calabria, Italy.

Burose, F., Anliker, T., Herd, D., Jungbluth, T. and Zäner, M. 2010. Readability of electronic ear tags in stationary antenna systems. Landtechnik 6: 446-449.

Caja, G., Conill, C., Nehring, R. and Ribò, O. 1999. Development of a ceramic bolus for the permanent electronic identification of sheep, goats and cattle. Comput. Electron. Agric. 24: 45-63.

Caja, G., Hernández-Jover, M., Conill, C., Garín, D., Alabern, X., Farriol, B. and Ghirardi, J. 2005. Use of ear tags and injectable transponders for the identification and traceability of pigs from birth to the end of the slaughter line. J. Anim. Sci. 83: 2215-2224.

Commission Decision of 15 December 2006 implementing Council Regulation (EC). No 21/2004 as regards guidelines and procedures for the electronic identification of ovine and caprine animals.

Council Regulation (EC). No 21/2004 of 17 December 2003 establishing a system for the identification and registration of ovine and caprine animals and amending Regulation (EC) No 1782/2003 and Directives 92/102/EEC and 64/432/EEC.

Dabbene, F. and Gay, P. 2011. Food traceability systems: Performance evaluation and optimization. Comput. Electron. Agric. 75: 139-146.

Dabbene, F., Gay, P. and Sacco, N. 2008a. Optimization of fresh-food supply chains in uncertain environments. Part I: Background and methodology. BioSyst. Eng. 99: 348-359.

Dabbene, F., Gay, P. and Sacco, N. 2008b. Optimization of fresh-food supply chains in uncertain environments. Part II: A case study. BioSyst. Eng. 99: 360-371.

Gay, P., Giuffrida, M. P., Piccarolo, P., Ricauda, A. D. and Tortia, C. 2007. Livestock and farm management improvement using RFID technologies. 32th CIOSTA Congress: Advances in labour and machinery management for a profitable agriculture and forestry, 17-19 Sep. 2007 Nitra, Slovakia. Book of Abstracts, 269.

Gay, P., Piccarolo, P., Ricauda, A. D. and Tortia, C. 2008. Livestock identification and farm management by RFID systems. AgEng-International Conference on Agricultural Engineering, Hersonissos, Crete, 23-25 Jun. 2008.

Ghirardi, J. J., Caja, G., Conill, C., Garín, D. and HernándezJover, M. 2004. Long term comparative trial of ear tags and ceramic boluses for the electronic identification of beef cattle under extensive conditions. ASAS-ADSA Joint Annual Meeting, ST. Louis, MO. 25-29 Jul. 2004.

Hessel, E. F., Reiners, K., Böck, S., Wendl, G. and van den Weghe, H. F. A. 2008. Application of high frequency transponders for simultaneous identification of weaned piglets. AgEng 2008 Congress: Agricultural \& Biosystems Engineering for a Sustainable World. Hersonissos, Crete, Jun. 23-25.

International Organization for Standardization. 1996a. Agricultural equipment. Radio-frequency identification of animals - Code structure. ISO 11784:1996 (E). 2nd ed. ISO, Geneva, Switzerland.

International Organization for Standardization. 1996b. Agricultural equipment. Radio-frequency identification of animals - Technical concept. ISO 11785:1996 (E). 1st ed. ISO, Geneva, Switzerland.
International Organization for Standardization. 2003. ISO 14223, 2003 ISO/IEC 14223: Radio frequency identification of animals - Advanced transponders. Part 1. ISO, Geneva, Switzerland.

International Committee for Animal Recording. 2005. Animal identification: List of manufacturer codes. [Online] Available: http://www.icar.org/manufacturer_codes.htm [2005 Dec. 09]. Klindtworth, M., Wendl, G., Klindtworth, K. and Pirkelmann, H. 1999. Electronic identification of cattle with injectable transponders. Comput. Electron. Agric. 24: 65-79.

Lambooij, E., van't Klooster, C. E., Rossing, W., Smits, A. C. and Pieterse, C. 1999. Electronic identification with passive transponders in veal calves. Comput. Electron. Agric. 24: $81-90$.

Liu, Y. and Shao, P. 2012. The application of RFID in the life-time traceability of animals. Int. J. Appl. Logistics 3: 54-65.

Leng, N. M., Kin, S. L., Hall, D. M. and Cole, P. H. 2005. A small passive UHF RFID tag for livestock identification. Proceedings of 2005 IEEE Interational Symposium on Microwave, Antenna, Propagation and EMC Technologies for wireless Communication, Beijing, China, August 2005.

Leong, K. S., Leng, N. M. and Cole, P. H. 2007. Investigation on the deployment of HF and UHF RFID tag in livestock identification. Proceedings of IEEE Antennas and Propagation International Symposium, Honolulu, HI.

Mutenje, T. J., Smithers, J. C. and Kgaphola, T. 2012. The development and evaluation of a radio frequency identification (RFID) based cattle handling system. Proc. CIGRAgeng 2012. 2012 Jul. 08-11. Valencia, Spain.

Mingxiu, Z., Chunchang, F. and Minggen, Y. 2012. The application used RFID in third party logistics. Phys. Procedia 25: 2045-2049.

Reiners, K., Hegger, A., Hessel, E. F., Böck, S., Wendl, G. and van den Weghe, H. F. A. 2009. Application of RFID technology using passive HF transponders for the individual identification of weaned piglets at the feed trough. Comput. Electron. Agric. 68: 178-184.

Ribó, O., Korn, C., Meloni, U., Cropper, M., De Winne, P. and Cuypers, M. 2001. IDEA: a large-scale project on electronic identification of livestock. Rev. Sci. Tech. Off. Int. Epiz. 20: 426-436.

Rossing, W. 1999. Animal identification: introduction and history. Comput. Electron. Agric. 24: 1-4.

Saa, C., Milá, M. J., Caja, G. and Ghirardi, J. J. 2005. Cost evaluation of the use of conventional and electronic identification and registration systems for the national sheep and goat populations in Spain. J. Anim. Sci. 83: 1215-1225.

Schwägele, F. 2005. Traceability from a European perspective. Meat Sci. 71: 164-173.

Smith, G. C., Pendell, D. L., Tatum, J. D., Belk, K. E. and Sofos, J. N. 2008. Post-slaughter traceability. Meat Sci. 80: 66-74.

Smith, G. C., Tatum, J. D., Belk, K. E., Scanga, J. A., Grandin, T. and Sofos, J. N. 2005. Traceability from a US perspective. Meat Sci. 71: 174-193.

Sydänheimo, L., Ydänheimo, M., Kivikoski, and Penttilä, K. 2006. Effect of item identifiers' length on passive radio frequency identification technology with supply chain applications. Int. J. Logistics: Res. Appl. 9: 143-156.

Thurner, S. and Wendl, G. 2007. Identification reliability of moving HF-transponders with simultaneous reading. Landtechnik 62: 106-107. 
Trevarthen, A. and Michael, K. 2007. Beyond mere compliance of RFID regulations by the farming community: a case study of the Cochrane dairy farm. IEEE Sixth International Conference on the Management of Mobile Business (ICMB), 9-11 July 2007, Toronto, ON.

Voulodimos, A. S., Patrikakis, C. Z., Sideridis, A. B., Ntafis, V. A. and Xylouri, E. M. 2010. A complete farm management system based on animal identification using RFID technology. Comput. Electron. Agric. 70: 380-388.

Wismans, W. M. G. 1999. Identification and registration of animals in the European Union. Comput. Electron. Agric. 24: 99-108. 